\title{
«Stellt Euch das mal im Kopf vor" - Wie Teaching Artists mit Schulklassen schreiben
}

\section{Christine Beckert, Mathilde Gyger und Franco Supino}

Im Jahr 2014 wirkten vier etablierte Autorinnen und Autoren im Vorfeld der Fussballweltmeisterschaft in Primarschulklassen der Nordwestschweiz als Teaching Artists: Sie leiteten im Projekt kick\&write ${ }^{\circledR}$ Schreibwerkstätten, deren Ziel es war, Kinder mit einer ungünstigen literalen Sozialisation zum Lesen und Schreiben zu motivieren. Sie wurden angespornt, einen Text zum Thema Fussball zu verfassen. Der Einsatz von Teaching Artists als Schreibcoaches ist eine spezielle schulische Fördermassnahme im Bereich der Literalität. Der Beitrag benennt die künstlerischen und (deutsch-)didaktischen Ziele und Konzepte der Teaching Artists und dokumentiert ihre Wahrnehmungen vom Schaffensprozess der Kinder. Die Steigerung der Lesemotivation gelingt an diesem non-formalen Bildungsort durch ungewohnte Zugänge zu Schriftlichkeit, durch Kompetenzerleben jenseits sprachformaler Normen, durch Anschlusskommunikation sowie durch enge Begleitung.

\section{Einleitung}

Das US-amerikanische Konzept Teaching Artist ist in Schulen im deutschsprachigen Raum mittlerweile bekannt. Es versucht, künstlerische Verfahren in einen pädagogischen Kontext zu integrieren, indem Teaching Artists - etablierte Kunstschaffende, etwa Musikerinnen und Musiker oder Theaterschaffende ihre Expertise auf dem Feld ihrer Kunst im Rahmen eines Projektes mit Kindern teilen.

Der vorliegende Beitrag stellt das Projekt kick\&write ${ }^{\circledR}$ vor, das im Rahmen einer fächerübergreifenden Zusammenarbeit von Sport- und Deutschdidaktik als Pilotprojekt im Frühjahrssemester 2014, im Vorfeld der Fussballweltmeisterschaft, realisiert wurde (Gramespacher et al., 2015). Der vorliegende Beitrag beschreibt die Arbeitsweise zweier Teaching Artists, die als Schreibcoaches im Rahmen des Projekts in Klassen des 5. Schuljahres im Einsatz waren. Er benennt ihre Ziele und dokumentiert, wie sie den Schaffensprozess in den Klassen beleuchten. 
Im deutschsprachigen Raum existieren unseres Wissens keine publizierten Evaluationen von Teaching Artist-Projekten. Empirisch gestützte Erkenntnisse sind entsprechend Desiderat. Dem vorliegenden Beitrag liegt eine explorative Fallstudie zugrunde. Die Ergebnisse beanspruchen keine Allgemeingültigkeit. Fragen nach der objektiv feststellbaren und längerfristigen Wirkung der Arbeit der Teaching Artists auf Lese- und Schreibmotivation, auf das literale Verhalten der Schülerinnen und Schüler sowie ihr Interesse an erzählender Literatur bleiben ausgeblendet.

\section{Teaching Artists als Vermittler Literarischen Schaffens}

Theoretischer Bezugsrahmen des vorliegenden Beitrags Teaching Artists als Kunst- und Kulturvermittler führen zur Begegnung und Zusammenarbeit mit einem schulfernen Experten. Dieser ist primär seinen eigenen Werten, Normen und Renommee als Autor oder Autorin verpflichtet. Als theoretischen Bezugsrahmen bei der Auseinandersetzung mit dem Teaching Artist (TA) als Vermittler literarischen Schaffens in Schulen schlägt der vorliegende Beitrag daher ein Verständnis von Literalität als kulturelle und soziale Praxis vor. Gemäss der Unterscheidung dreier Dimensionen von Literalität nach Rosebrock \& Bertschi-Kaufmann (2013) nimmt ein Verständnis von Literalität als «kultureller und sozialer Praxis» weniger normative schulische Konzepte beziehungsweise auf diese bezogene individuelle Kompetenzen in den Blick als «alltagskulturell[e]» und ausserschulische Aspekte (etwa literale Praktiken oder Sozialisationsverläufe) und diskutiert deren Bedeutung für schulische Schreibkultur (S. 11).

Die Leithypothese für die explorative Begleitforschung zum TA ist einerseits diesem Verständnis verpflichtet. Andererseits nimmt sie die von Bachmann und Becker-Mrotzek (2010) formulierte Position auf, dass Schreibaufgaben jeglicher Art, die für den Schreibprozess und die Schreibentwicklung förderlich sein sollen, «den Lernprozess in einen authentischen und sozialen Kontext einbetten, in dem das Schreiben einen für die Schülerinnen und Schüler erkennbaren Sinn bekommt» (S. 191). Die beiden Autoren formulieren vier Bedingungen «sinnvoller schreibdidaktischer Settings» (S. 195): (a) Der Text sollte eine «identifizierbare Funktion erfüllen», was einschliesst, dass Ziel und Adressaten bekannt sind; (b) Das Setting sollte Gelegenheit zur Aneignung sprachlichen, kultur- bzw. schreibtechnischen Wissens und Weltwissens bieten; (c) in einen Kontext sozialer Interaktion eingebettet sein und (d) eine Auseinandersetzung mit der Wirkung des Textes ermöglichen.

Die leitende Hypothese des vorliegenden Beitrags besteht in der Annahme, dass TAs den Schülerinnen und Schülern, mit denen sie arbeiten, solchermassen situierte Schreib- und Leseerfahrungen ermöglichen. Im Unterschied 
zu der explorativen Studie von Bachmann \& Becker-Mrotzek (2010) zielt der vorliegend Beitrag nicht darauf ab, die Wirksamkeit einer situierten Schreibund Leseerfahrung darzustellen, sondern ein bisher kaum untersuchtes schreibdidaktisches Setting anhand der oben formulierten Kriterien zu beschreiben. In diesem schulischen Setting findet unter Anleitung und Beteiligung der Schreibcoaches ein Prozess statt, bei dem davon auszugehen ist, dass er den Grundsätzen des kooperativen Schreibens (Lowry, Curtis \& Lowry, 2004) folgt.

\section{Begriff und Konzept des Teaching Artist}

Obwohl der Einbezug von Kunstschaffenden in Schulen in den USA eine lange Tradition hat, wurde der Begriff des Teaching Artist erst 2003 mit der Gründung des Teaching Artist Journal (Booth, 2003) geprägt. Dem Konzept des TA liegt die Überzeugung zugrunde, dass die Produktion von Kunst lernbar ist und dass es sinnvoll ist, etablierte Kunstschaffende zur Vermittlung künstlerischen Schaffens in Schulen einzusetzen. Kunstschaffende werden als TAs in der Regel projektbezogen in den Schulalltag integriert, unterstützt von einer Lehrperson, die begleitend organisatorische Aufgaben wahrnimmt. Träger von TA-Projekten sind in der Regel schulische oder ausserschulische kulturpädagogische Einrichtungen. TAs qualifizieren sich für solche Projekte durch ihre erfolgreiche, d.h. gesellschaftliche Resonanz erfahrende, künstlerische Tätigkeit. Sie geben Einblick in ihren kreativen Arbeitsprozess, ihre professionellen Ansprüche und vermitteln ihre eigenen Problemlösungsstrategien. Die Schülerinnen und Schüler, mit denen TAs arbeiten, werden in den gesamten künstlerischen Schaffensprozess eingebunden (Booth 2009).

\section{Begleitforschung zu Teaching Artists}

Bislang existiert eine einzige, aber umfangreiche Untersuchung zur Arbeit der TAs: die US-amerikanische Studie zum «Teaching Artist Research Project» (Rabkin, Reynolds, Hedberg \& Shelby, 2011) der University of Chicago. Zwischen 2008 und 2011 wurden über 3550 Künstlerinnen und Künstler interviewt, die in 12 US-Bundesstaaten in Teaching-Artist-Projekten involviert waren. Die Studie zielt auf eine verbesserte Steuerung der künftigen Ausrichtung solcher Programme ab. Die Empfehlungen sind vielfältig. Sie fordern eine bessere Triangulation zwischen Klassenlehrer, Fachlehrer (z.B. Instrumentallehrer) und dem TA ein und betonen die Notwendigkeit weiterer und vertiefter Forschung (Rabkin et al., 2011). Die Studie hält fest, dass sich Schulen mit dem Einbezug von TAs ausserschulisch entwickelte künstlerische Ressourcen zunutze machen, was auf Seiten der TAs eine hybride Identität voraussetzt (Rabkin et al., 2011, S. 45):

The dual or hybrid character of teaching artists makes their lives challenging. They seem, in some respects, to fall between the two fields - the arts and education - and their work is not fully validated or valorized by either. But it is their hybridity that can make them uniquely powerful educators. 
Projekte der Literaturvermittlung in der Schweiz

Die Berner Fachhochschule bietet seit dem Studienjahr 2011/12 einen CAS Teaching Artist für professionell arbeitende Kunstschaffende an. Die bisher im Rahmen des Studiengangs entwickelten Projekte und auf der Webseite dokumentierten 28 Projekte sind mehrheitlich im Bereich der bildenden Kunst, in Musik, Tanz, Theater oder Film angesiedelt mit der Ausnahme zweier interdisziplinärer Projekte unter Beteiligung kreativen Schreibens und zweier Schreibwerkstätten (Hochschule der Künste Bern, 2017).

Im Bereich der Literaturvermittlung sind in der Schweiz die klassischen Autorenlesungen weit verbreitet. Zu nennen sind etwa die Projekte in Zürich und in der Zentralschweiz, die unter dem Label Literatur aus erster Hand jährlich Hunderte von Schullesungen im Klassenzimmer ermöglichen (Volksschulamt Kanton Zürich, 2017).

Daneben sind in den letzten Jahren weitere innovative literarische Projekte entstanden: Der Schweizer Schriftsteller Richard Reich entwickelte gemeinsam mit der österreichischen Germanistin und Lektorin Gerda Wurzenberger das Projekt Schulhausroman, das seit 2005 erfolgreich versucht, sprachlich weniger versierte Schülerinnen und Schüler zwischen 12 und 18 Jahren zu erreichen (Die Provinz, Kulturprojekt, 2017). Unter Anleitung von Schreibcoaches sind in den letzten Jahren über 50 Schulhausromane in Schulen in der ganzen Schweiz entstanden. Inzwischen wird dieses Konzept auch in Deutschland und Österreich umgesetzt.

Als "Autorin in Schul-Residenz» begleitet und initiiert die Schriftstellerin Dragica Rajcic während drei bis vier Halbtagen pro Woche in einem ausgewählten Schulhaus in enger Zusammenarbeit mit Lehrpersonen Sprach- und Literaturprojekte (Artlink Büro für Kulturkooperation, 2017). Dabei werden den beteiligten Schülerinnen und Schüler u.a. Schreib- und Leseerfahrungen in ihrer Herkunftssprache ermöglicht.

Ausserdem ist auch auf das Programm der Aargauer Kulturvermittlung Kultur macht Schule hinzuweisen, in dessen Rahmen Workshops und Schreibwerkstätten mit Autorinnen und Autoren angeboten werden (Kulturvermittlung Kanton Aargau, 2017).

Projektevaluationen - so solche existieren sollten - sind leider nicht zugänglich. Mit dem Beitrag von Bürki \& Stucki-Volz (2010) liegt eine schreibdidaktische Reflexion eines kooperativen Schreibprojekts im 10. Schuljahr vor, das zu einem Klassenroman führte, allerdings ohne Beteiligung eines Schreibcoaches. 


\section{Das Teaching Artist-Projekt kick\&write ${ }^{\circledR}$}

\section{Projektanlage}

kickerwrite $^{\circledR}$ zielt als die Fächer Deutsch und Sport verbindendes schulisches Projekt darauf ab, Kinder sowohl im Umgang mit Fussball als auch mit Literatur und insbesondere in Bezug auf das Schreiben eigener erzählender Texte zu fördern. Äusserer Anlass für das Pilotprojekt zu kick\&write ${ }^{\circledR}$ war die Fussballweltmeisterschaft im Jahr 2014.

Zwei Autorinnen und zwei Autoren stellten sich der Aufgabe, im Verlauf von zwei Monaten mit jeweils einer Klasse im 5. Schuljahr in insgesamt acht halbtägigen Schreibwerkstätten eine Geschichte zum Thema Fussball zu verfassen, die anschliessend in einem gegenseitigen peer review-Verfahren von den anderen beteiligten Klassen kriteriengeleitet bewertet und in einem Sammelband publiziert wurde (Alves, Blume, Bortlik \& Hughes, 2014).

Im ersten Teil des Projekts (Februar bis April 2014) erarbeiteten die Klassen ihren Text. In der zweiten Phase (Mai bis Juni 2014) lasen sie die drei Geschichten der anderen Klassen und bewerteten sie anhand eines vorgängig mit den TAs diskutierten Kriterienrasters. Grundlage des Rasters waren die Überlegungen von Leubner, Saupe \& Richter (2010). Am Ende des Schuljahres folgten die Publikation der Geschichten, Kurzlesungen im Literaturhaus Basel und die Prämierung des Siegertextes. Abgesehen von dieser zeitlichen und organisatorischen Rahmung und der nicht näher bestimmten thematischen Vorgabe (Fussballgeschichte), liess die Projektanlage den TAs Freiheit zur individuellen Ausgestaltung ihrer Arbeit mit den Klassen.

Da erst wenige Studien zur Arbeit von TAs existieren, wurde das Projekt von explorativer Forschung begleitet, mit dem Ziel, weiterführende Fragestellungen und Desiderate zu formulieren. Die Begleitforschung zur Arbeit der TAs führte zu Einzelfallanalysen, die im Folgenden entlang theoriebasierter Fragestellungen präsentiert werden.

\section{Fragestellungen der explorativen Begleitforschung}

Wenn aufgrund der leitenden Hypothese (s. Kap. «Theoretischer Bezugsrahmen des vorliegenden Beitrags») davon auszugehen ist, dass der TA einer Klasse das Angebot lernförderlicher Schreib- und Leseerfahrung ermöglicht (Bachmann \& Becker-Mrotzek, 2010), dann schliessen sich daran folgende vier Fragestellungen an:

1. Erfüllt das Angebot eine erkennbare kommunikative Funktion?

2. Bietet es Gelegenheit zur Aneignung sprachlichen, kultur- bzw. schreibtechnischen Wissens und Weltwissens und wenn ja inwiefern?

3. Verlangt es nach sozialer Interaktion und wie gestaltet sich diese?

4. Führt es zu einer Auseinandersetzung mit der Wirkung von Texten und wenn ja, inwiefern? 
Nachfolgend wird versucht, Antworten auf diese Fragen anhand der Aussagen und Reflexionen zweier ${ }^{1}$ (von insgesamt vier am Projekt beteiligten) TAs zu finden, um die Hypothese und die mit ihr verbundenen Fragestellungen im Hinblick auf weiterführende Studien zu schärfen und ggf. zu erweitern.

\section{Forschungsdesign}

Für die explorative Begleitstudie wurden Daten zur Arbeit aller vier am Projekt beteiligten TAs erhoben, um auf dieser Grundlage Einzelfallanalysen zu erstellen. Die Einzelfallanalysen beruhen auf Daten aus Grobplanungen, Logbüchern und vor allem aus Experten-Interviews mit den TAs:

- Grobplanungen: Die TAs äusserten sich in einer vorgängigen Grobplanung zu den einzelnen Phasen des Projektverlaufs, zu ihren eigenen Zielen und Aktivitäten sowie zu den Aktivitäten der Schülerinnen und Schüler und zu den verwendeten Medien. Aus den Grobplanungen wurden bei der Analyse gezielt Inhalte berücksichtigt, die im Experteninterview und/oder den Logbüchern erfolgte Aussagen differenzieren, ergänzen oder ihnen eindeutig widersprechen.

- Logbücher: Während des Projekts dokumentierten die TAs den Fortgang der Arbeit in einem Logbuch. Zweck der Logbücher war es, die Planung und Gestaltung des Prozesses und den Einbezug der Kinder zu dokumentieren. Die TAs wurden gebeten, das Logbuch einmal pro Woche und zwar möglichst direkt im Anschluss an das jeweilige Arbeitstreffen mit der Klasse auszufüllen und an die Projektleitung zu senden. Insofern gewähren diese Daten Einblick in die unmittelbar nach der Schreibwerkstatt entstandene Wahrnehmung der TAs.

- Halbstrukturierte Experteninterviews (kurz nach Projektende): Ziel war es, die TAs im Rückblick eine Gesamteinschätzung des Projekts vornehmen zu lassen und die Arbeit zu reflektieren. Zudem sollten die Aussagen in Grobplanung und Logbüchern gespiegelt werden. Der Interviewleitfaden war grob in folgende Bereiche gegliedert: Konzepte zu Lesen, Schreiben und Lese-Schreib-Unterricht; Werte und Ansprüche der TAs als Literaturschaffende; Einschätzung der Arbeit der Kinder; Raum für individuelle inhaltliche Schwerpunkte und Ausführungen.

Die Auswertung der Experteninterviews folgte einer theoriegeleiteten, kombiniert induktiv-deduktiven Vorgehensweise. Analysemethode war die sozialwissenschaftliche qualitative Inhaltsanalyse nach Mayring (2003). Diese erlaubt es, festgehaltene Kommunikation «systematisch» und «theoriegeleitet» zu analysieren (Mayring, 2003). Im Rahmen dieser Inhaltsanalyse wurden zum einen Haupt- und Unterkategorien gebildet, die sich aus den Fragestellungen des Projekts ergaben. Zum anderen wurden Kategorien gebildet, welche sich auf die von den TAs zusätzlich eingebrachten Aspekte bezogen. Ziel dieses Vorgehens war es, zu einem vertieften Verständnis des Vorgehens zu gelangen und dieses deskriptiv und zusammenhängend darzustellen. 
Die Daten aus diesen Dokumenten wurden in der Auswertung trianguliert. In der nun folgenden Ergebnisdarstellung sind die Dokumente wie folgt gekennzeichnet: Verweise auf die Grobplanung, mit ihren Teilen «A. Grobkonzept und Ziele» und «B. Verlaufsplanung», erfolgen mit (GP, Teil A bzw. B), Verweise auf die Logbücher mit (LB, Tag. Monat. Jahr, Fragenummer; z.B. LB, 23.5., 1.2 für «Logbuch des 23. Mai 2014, Frage 1.2»), und Verweise auf das Experteninterview mit (EI, Tag. Monat. Jahr).

Die Experteninterviews mit den Autorinnen und Autoren führten zu qualitativ und quantitativ vergleichbaren Daten; bei den in den Grobplanungen und Logbüchern von den TAs erfassten Daten gab es hingegen wie zu erwarten beträchtliche individuelle Unterschiede. Bei der Auswertung wurden alle gesammelten Daten berücksichtigt. Zwei für die leitenden Fragestellungen aufschlussreiche Fallbeispiele wurden schliesslich zu der folgenden Ergebnisdarstellung verarbeitet.

\section{Ergebnisdarstel lung}

Nachfolgend werden die Angebote der beiden ausgewählten TAs entlang der im Kapitel «Fragestellungen der explorativen Begleitforschung» formulierten Fragestellungen im Kontext der Projektanlage beschrieben und mit kurzen Auszügen aus den analysierten Dokumenten veranschaulicht. Die beiden TAs sind anonymisiert als Kickfuchs bzw. Ballfee bezeichnet.

Kommunikative Funktion der Texte. Die Projektanlage gab vor, dass in der ersten Arbeitsphase mit den TAs in jeder Klasse eine literarische Erzählung oder Kurzgeschichte zum Thema Fussball im weitesten Sinn entstehen sollte. Die Geschichten wurden in der zweiten Projektphase in einem peer review-Verfahren von den anderen Klassen gelesen und bewertet und im Juni 2014 kurz vor Abschluss des Projekts publiziert. Die beste Erzählung wurde prämiert. Funktion, Ziel und Adressaten der Texte waren damit vorgegeben. Den TAs ging es auf diesem Hintergrund darum, bei den Schülerinnen und Schüler vor allem eine produktive Grundhaltung gegenüber dem literarischen Schreiben zu fördern.

Ballfees Projektziel war es, "den Mut, das Gross-und-weit-Denken»(EI, 19.06.2014) der Schülerinnen und Schüler und die Freude am literarischen Schreiben zu fördern, damit sie gute Schreiberinnen und Schreiber würden. Ein guter Schreiber ist nach ihrer Auffassung:

Jemand, der das wirklich total von innen heraus und mit Freude und Engagement macht. Also keine Kriterien ---. Egal, ob jetzt dieser Buchstabe vor oder nach jenem kommt. Schreiben, schreiben, Freude daran haben. Dass es etwas Kreatives ist. Und wo man etwas von sich reingeben kann. Etwas erschaffen. (EI, 19.06.2014) 
Ballfee bezeichnete die Freude der Schülerinnen und Schüler am Schreiben und Vorlesen des Geschriebenen als Grund für ergiebiges und erfolgreiches Arbeiten (z.B. LB, 20.2., 1.2.c). Sie zeigte sich beeindruckt vom Ideenreichtum in der Klasse, mit der die arbeitete, der völligen Hingabe beim Schreiben wie beim Fussballspielen. Nichts sei schwierig zu realisieren gewesen, «die Kinder [sprudelten/schrieben], die Zeit raste» (z.B. LB, 13.2., 1.4.b).

Kickfuchs lag es primär daran, die Denkprozesse der Schülerinnen und Schüler anzuregen. Dies erforderte die Bereitschaft zum «Eingehen auch auf die wildesten und unbrauchbarsten Ideen» (LG, 25./26.3., 1.4.a):

Das war wirklich ganz toll [...] den Denkprozess, den ich gesehen [...] ich habe immer gesehen, wie es in den kleinen Köpfen arbeitet, und habe versucht, das irgendwie rauszukitzeln. Das ging sehr gut, weil es die Kinder selber immer gesehen haben. (EI, 14.06.2014)

Bei Kickfuchs war der Schreibprozess jedoch letztlich vom inhaltlichen Anspruch geleitet, eine klare, d.h. realitätsnahe Geschichte zu entwerfen, welche auf Absurdes bzw. Surreales verzichtete und logisch einleuchtend war (EI, 14.06.2014). Kickfuchs betonte jedoch, er sei immer sehr auf die Kinder eingegangen, habe nicht «diktatorisch" (EI, 14.06.2014) etwas durchsetzen wollen. So liest man denn in einem der letzten Logbücher nicht nur von neuen Ideen für den Schluss, sondern auch: "Vorschläge von mir, die Geschichte anders zu gestalten, wurden abgelehnt, [weil] die Klasse es so beschlossen hat!» (LB, 29./30.4., 1.3).

Gelegenheit zur Aneignung von Wissen. Die gemeinsame Arbeit am Erzähltext erforderte aus Sicht der TAs seitens der Schülerinnen und Schüler eine intensive Auseinandersetzung mit Aspekten von Textstruktur, Handlungslogik, Figurengestaltung und Wortwahl. Dabei sollten die Schülerinnen und Schüler einen grossen Teil des Textproduktionsprozesses miterleben und mitgestalten.

Ballfee begann die Arbeit mit der Frage an ihre Klasse, was eine gute Geschichte ausmacht. Die Auseinandersetzung zu dieser Frage erfolgte anhand von selbst mitgebrachten Lieblingsgeschichten der Kinder und mündete in eine konsensfähige Auswahl von Kriterien. Im Anschluss ging es an die Wahl und Charakterisierung der Hauptfigur. Die folgende Schreibwerkstatt war zunächst der kritischen Würdigung verschiedener möglicher, von Ballfee auf der Grundlage der notizenhaften Beschreibungen der Kinder vertexteten Ausgestaltungen der Hauptfigur gewidmet. Dann wurde eine dieser Ausgestaltungen definitiv gewählt. Zudem wurden Ort und Zeit der Handlung bestimmt sowie erste Ideen für die Handlung skizziert. Die dritte Schreibwerkstatt setzte mit der von Ballfee verfassten Einleitung der Geschichte als Reflexionstext ein; anschliessend wurde die Handlung weiterentwickelt. In nachfolgenden Treffen entstanden allmählich die beiden Hauptteile der Geschichte; dabei orientierten sich TA und Klasse an der Grobstruktur von Erzählungen (Problem - Aufgabe - Hindernisse - Lösung, nach Claussen \& Merkelbach, 1995) und tauschten Änderungs- 
und Verbesserungsvorschläge aus. Bei der Beurteilung vorliegender Textteile knüpfte Ballfee an die in der Klasse bereits etablierte Argumentationskultur an und forderte von den Kindern "knallhart» (EI, 19.06.2014) Begründungen für Urteile (bezüglich der Qualität der Geschichte). Unbegründete Urteile liess sie nicht zu. Hinsichtlich der Textlogik unterstützte Ballfee die Schülerinnen und Schüler, indem sie Vergleiche zur Handlungslogik von Filmen herstellen liess. Wiederholt betonte sie die Argumentationsbereitschaft der Kinder, etwa bezüglich Realitätsgehalt der Geschichte oder in der Besprechung neu vorgelegter Textteile. Auch erstaunte sie die Urteilsschärfe der Schülerinnen und Schüler, z.B. bei der Beurteilung des von ihr geschriebenen Geschichtenanfangs: "pointiert in Lob und Tadel, mit klaren Vorstellungen, was sie gut finden und was nicht (zum Beispiel die Schimpfwörter muss ich streichen)» (LB, 20.2., 1.3).

$\mathrm{Zu}$ Beginn der Arbeit liess sich auch Kickfuchs über die literarischen Vorlieben der Kinder informieren. In einer Diskussion erfolgte dann die Wahl der Genres für die Geschichte: Liebesgeschichte und Krimi. Nach der Wahl des Genres war die zweite Schreibwerkstatt einen knappen Monat später der Struktur sowie dem Handlungsentwurf gewidmet.

Kickfuchs war daran gelegen, dass die Schülerinnen und Schüler Logik und Plausibilität des Handlungsverlaufs erkennen und mit der Verständlichkeit des Textes in Verbindung bringen konnten. Bisweilen wies er konkret auf logische Unstimmigkeiten einzelner Handlungselemente hin (EI, 14.06.2014). Ging es darum, an der Logik des Textes zu arbeiten, erfolgte die Aufforderung: «Stellt euch das mal im Kopf vor, stellt euch das wie einen Film vor, was hier abläuft, schliesst die Augen» (EI, 14.06.2014). Im Bereich der Handlungslogik habe er, immer auf der Ebene von Planung und Reflexion, sehr stark in die Entstehung der Geschichte eingegriffen; so wurde für die Kinder auch ersichtlich, dass eine gute Geschichte nichts «Hochfliegendes» (EI, 14.06.2014) braucht.

Ein weiterer Schwerpunkt war die Figurenbeschreibung, für ihn ein wesentliches Moment der Spannungserzeugung, wie «eine Boje» (EI, 14.06.2014) für die Weiterentwicklung der Geschichte. Kickfuchs brachte den Kindern nahe, nicht die Äusserlichkeiten von Figuren zu beschreiben, sondern sie handeln zu lassen und so zu charakterisieren. Seine wiederholte Anregung lautete: «Überlegt euch: Was macht eine Figur interessant? Das ist doch, wie sie redet, was sie macht, wie sie aussieht» (EI, 14.06.2014).

Kickfuchs führte aus, seine nachdrücklichen Hinweise auf die Bedeutung der Handlungslogik hätten insofern Wirkung gezeigt, als die diesbezüglichen Diskussionen der Kinder immer pragmatischer geworden seien; zudem nahm er im Projektverlauf bei diesem Textaspekt eine deutliche Verbesserung wahr. Die Wirkung seiner Anregungen bezüglich Figurengestaltung und seine Hinweise auf die Bedeutung von Handlungsbeschreibungen für die Figurencharakterisierung sah Kickfuchs darin, dass die Kinder von sich aus zunehmend mit direkter Rede arbeiteten, die Figuren somit empfehlungsgemäss handeln liessen, anstatt sie bloss zu beschreiben. 
Neben den eigenen Schwerpunkten nahm Kickfuchs auch Anliegen der Schülerinnen und Schüler auf, z.B. die konkrete Wortwahl: Er schlug jeweils Begriffe vor und versicherte sich, dass diese für die Kinder verständlich waren. Bei der Rohfassung des Gesamttextes wurde viel Zeit verwendet, um an einzelnen Formulierungen zu arbeiten (EI, 14.06.2014).

Soziale Interaktion. Beide Geschichten entstanden in einem Wechsel von gemeinsamer Partner- oder Gruppenarbeit beim Verfassen von Textfragmenten und Plenumsdiskussionen über vorliegende Textteile. Der Schreibprozess verlief bei beiden TAs iterativ: Die Kinder erhielten die aus der Ausarbeitung bzw. der Redaktion der resultierenden Textteile zur kritischen Würdigung; daraus entwickelten sie jeweils neue Ideen für weitere Textbausteine, den weiteren Handlungsverlauf und die einzelnen Figuren betreffend. Die geplanten Vorgehensweisen wurden praktisch ohne Kursänderung oder gar Bruch realisiert, sodass ein Ganzes von Planung, Steuerung und Schreibcoaching entstand. Beide TAs regten einen kooperativen Schreibprozess an, in den sie sich jedoch auf unterschiedliche Art einbrachten. Ballfee erstellte von vornherein eine detaillierte Planung und verarbeitete die Ideen der Kinder selbst zu einem Text. Kickfuchs zog ein grob geplantes und spontanes Vorgehen vor und liess die Kinder die Geschichte selbst schreiben. Er verband die Textteile und redigierte sie.

Für Ballfee war bereits vor Projektbeginn klar, dass sie die Geschichte selber schreiben würde: «In meiner Rolle als Autorin bin ich für das Schreiben zuständig. Lieferantinnen und Lieferanten für den Erzählstoff sind die Kinder.» (GP, Teil A). Ballfees Planung sah während der Projektarbeit einen sich über die Schreibwerkstätten hinweg wiederholenden Rhythmus vor: Entgegennehmen der Ideenentwürfe der Kinder und deren Vertextung zur Geschichte, die Reaktion der Kinder auf die Vertextung und aus der entsprechenden Diskussion resultierende neue Schreibziele, welche die nächste Schreibphase der Kinder bestimmten (GP, Teil B). Die Ideen der Kinder in den in Partnerarbeit verfassten Textteilen verarbeitete Ballfee zu ihrem eigenen Text. Der Text basierte somit weitestgehend auf den Ideen, nicht aber den Formulierungen der Kinder. In einer gemeinsamen Besprechung wurde geklärt, ob die Kinder ihre Ideen in Ballfees Text wiedererkannten und mit diesem einverstanden waren (LB, 28.3., 1.2.c). Ein Logbucheintrag gibt Aufschluss über entsprechende zentrale Fragen: «Ihre [die Entwürfe der Kinder] skizzierten Figuren, die der Coach in Sätze gefasst hat: Was haben die Kinder gedacht, empfunden beim Lesen? Haben sie ihre Figuren wiedererkannt? Weitergedacht?» (LB, 13.2., 1.1).

Nur in geringem Ausmass, nämlich bei den Figurenbeschreibungen zu Beginn der Geschichte, liess Ballfee Formulierungen der Kinder einfliessen. Diese Vorgehensweise war für Ballfee die einzig denkbare Form des Schreibprozesses. Rückblickend meinte Ballfee, sie habe die Kinder stark geführt - im Rahmen ihrer genauen Planung und deren flexibler Anpassung an Vorlieben und Verhalten der Kinder, durch eine genaue Strukturierung des Gesamtpro- 
zesses und das Rhythmisieren einzelner Arbeitsphasen (EI, 19.06.2014).

Kickfuchs hatte in der Vorbereitung keinen klaren Plan erstellt, sondern grobe Leitlinien gesetzt. Er entschied sich für ein spontanes, intuitives Vorgehen, das es ihm erlaubte, situativ auf Reaktionen in der Klasse einzugehen (EI, 14.06.2014). Angesichts des knappen Zeitrahmens, den es mit einer unbekannten Klasse einzuhalten galt, sollte die Geschichte bausteinartig, also im fortwährenden Aufbau auf Vorhandenem, entstehen. Die Nutzung dieses Baukastenprinzips bot Strukturierung und Rhythmisierung beim Schreiben und erlaubte, «einfach aufeinander[zu]stellen, was kommt» (EI, 14.06.2014). Aus der Besprechung und Beurteilung vorliegender Textteile ergaben sich fortwährend neue Schreibaufträge. In Plenumsarbeit wurde das genreorientierte Grobschema der Geschichte entwickelt, mit den Figuren für die - gemäss klarem Wunsch der Klasse autobiographische Fussballgeschichte. Fortan arbeiteten die Kinder in Arbeitsgruppen. Die Leitenden der sechs Gruppen wurden von Kickfuchs bestimmt; diese durften sich ihre Teams von 3-4 Kindern zusammenstellen (LB, 25./26.3., 1.1). Jede Gruppe wählte ihren Auftrag, nämlich einen Handlungsstrang bzw. ein Thema zu verfolgen. Ein Kind schrieb, aber alle erfüllten in Kooperation den Auftrag und konnten dem TA schliesslich einen gemeinsam verantworteten Entwurf vorlegen. Der TA verband am Computer die in den Gruppen entstandenen Teiltexte und verbesserte gegebenenfalls im Hinblick auf Rechtschreibung und Wortwahl. Entdeckte er beim Zusammensetzen der Textteile Lücken oder Unstimmigkeiten, ergaben sich Aufträge für die Arbeitsgruppen. Bei konkurrierenden Ideen erfolgte eine Plenumsdiskussion mit Abstimmung. Zwar versuchte Kickfuchs jeweils, die aus seiner Sicht «bessere» Idee zu stützen, akzeptierte aber auch Ideen, die ihm persönlich nicht gefielen.

Auseinandersetzung mit der Wirkung von Texten. Die Entwicklung einer eigenen Fussballgeschichte und die Beurteilung der Geschichten der drei anderen Projektklassen boten Gelegenheit zur Auseinandersetzung mit der Wirkung der Geschichten.

Ballfee hebt hervor, die Schülerinnen und Schüler hätten über klare Kriterien verfügt, was eine spannende Geschichte ausmache. Als Gründe für erfolgreiches Arbeiten nannte sie "die differenzierte Wahrnehmung und Kritikfreudigkeit [...] der Kinder», deren «[...] Fähigkeit [...], ihre Überlegungen in präzise Voten zu fassen und der Mut, sie auch vor allen zu vertreten» (z.B. LB, 16.5., 1.2.c). An Überraschendem vermerkte sie «die pointierten Fragen, zum Beispiel: ,Wie machen wir es, wenn wir den Ort des Geschehens wechseln? Was passiert mit der Ich-Figur?" "(LB, 27.2., 1.3), und die Diskussionen der Kinder würdigend: «Die Kinder sind sehr differenziert und kritisch, haben automatisch mit dem Lektorat begonnen, indem sie Unstimmigkeiten im Text aufdeckten" (z.B. LB, 3.4., 1.2.a). Den beachtlichen Reflexionsfähigkeiten entsprächen indes noch nicht die für die Umsetzung notwendigen Schreibfähigkeiten; hier erkannte Ballfee grossen Entwicklungsbedarf. 
Kickfuchs äusserte sich vor allem zu Schwierigkeiten, z.B. der «Vermittlung, was eine Geschichte ist bzw. sein kann. Mehrere Motive, mehrere Handlungsstränge können sich die Schülerinnen und Schüler (noch) nicht vorstellen» (LB, 25.2., 1.2.b und c), oder die «Vermittlung gewisser gestalterischer Kniffe. Detektivgeschichte, Logik der Handlung. Möglicherweise sind Schülerinnen und Schüler überfordert» (LB, 20.3., 1.4.b und c). Auch die Textbeurteilung war für die Kinder mühevoll: «Die Kinder sind es nicht gewöhnt, detaillierte Beurteilungen abzugeben» (LB, 20.5., 1.2.b und c), sie neigten laut Kickfuchs zu eher allgemeinen Urteilen (z.B. «die Geschichte ist furchtbar langweilig», EI, 14.06.2014). Kickfuchs nannte einige von den Kindern angewendete Kriterien: keine Schimpfwörter verwenden, mangelnde Figurenzeichnung, merkwürdiges Einführen älterer Kinder bzw. Jugendlicher, Fehlen des Fussballmotivs (LB, 20.5., 1.2.b).

\section{Diskussion}

Das Wirken von TAs im Klassenzimmer, im Projekt kickerwrite ${ }^{\circledR}$ von professionellen Literaturschaffenden, schafft eine aussergewöhnliche Situation. Die Schülerinnen und Schüler erhalten Gelegenheit, einen künstlerischen Schaffensprozess unmittelbar mitzuerleben und mitzugestalten. Dabei sind sie mit den künstlerischen Arbeitsformen und mit den spezifischen Schwerpunkten, Orientierungen und Wissensbeständen der jeweiligen TAs konfrontiert.

Die leitendende Hypothese dieses Beitrags geht davon aus, dass der TA einer Klasse das Angebot lernförderlichen Schreib- und Leseerfahrung ermöglicht (s. 2.1) und formuliert nach Bachmann \& Becker-Mrotzek (2010) vier Fragestellungen zu einem solchen Setting. Der Einblick in die konkrete Arbeit zweier TAs anhand der Dokumentationen und Reflexionen zeigt, dass diese Annahme im Fall dieser beiden analysierten Angebote berechtigt ist:

1. Die kommunikative Funktion und die Zielsetzung des Schreibangebots war durch die Projektanlage vorgegeben. Aus Sicht der beiden TAs gelang es, Schreibfreude und Denkprozesse der Schülerinnen und Schüler anzuregen und eine produktive Grundhaltung für das literarische Schreiben zu fördern.

2. Die Schülerinnen und Schüler sollten einen grossen Teil des Textproduktionsprozesses miterleben und handelnd mitgestalten. Gelegenheiten zur expliziten Wissensaneignung bestanden in den Bereichen von Handlungsstruktur und -logik, Figurengestaltung und Wortwahl.

3. Die soziale Interaktion beim Schreiben bestand aus einem unterschiedlich gestalteten Wechsel von kooperativer Partner- oder Gruppenarbeit beim Verfassen von Textfragmenten und Plenumsdiskussionen über vorliegende Textteile. Ballfee sah sich in ihrer Rolle als Autorin explizit für das Schreiben der Geschichte zuständig und verarbeitete die Ideen der Schülerinnen und 
Schüler zu einem ausformulierten Text, dessen einzelne Bausteine sie mit der Klasse diskutierte. Kickfuchs interpretierte seine Rolle als Schreibcoach weitgehend als Redakteur und Lektor der von den Kindern verfassten Textbausteinen. Er liess die Kinder die Geschichte primär selbst schreiben, redigierte die Texte, fügte sie zusammen und diskutierte allfällige Unstimmigkeiten im Handlungsverlauf mit der Klasse.

4. Die Entwicklung einer eigenen Fussballgeschichte und die Beurteilung der Geschichten der drei anderen Projektklassen als vorgegebene Elemente der Projektanlage und als Basis des Literaturwettbewerbs boten Gelegenheit zu einer intensiven Auseinandersetzung mit der Wirkung der Geschichten.

Die Arbeit am Verständnis von kommunikativer Funktion und Zielsetzung der Texte war durch eine spezifische Rahmung mit Literaturwettbewerb und Publikation und einem entsprechend definierten Auftrag bestimmt. Wie Bürki und Stucki-Volz (2010) ausführen, kann ein Endprodukt in Form eines gebundenen Buches für die Schreibenden von grosser Bedeutung sein. Da die spezifische Situiertheit einer schreibdidaktischen Aufgabe mit dafür ausschlaggebend ist, wie die Lernenden die Aufgabenstellung angehen und bearbeiten (Bachmann \& Becker-Mrotzek 2010), ist davon auszugehen, dass andersartige Vorgaben die Zielsetzung auch aus Sicht der beteiligten TAs modifiziert hätten. Ob es auch ohne den Ansporn von Publikation und Wettbewerb zu einer vergleichbar intensiven Auseinandersetzung mit Textqualität und Textwirkung kommen kann, bleibt zu untersuchen. Die kooperative Entwicklung von Handlungsstruktur und -logik, Figurengestaltung und Wortwahl waren im Fall von kickerwrite ${ }^{\circledR}$ Schwerpunkte der Förderung, während die Arbeit in bestimmten Bereichen (Ausformulierung oder sprachformale Überarbeitung) von den TAs übernommen wurde. Es stellt sich die Frage, ob es nicht möglich wäre, auch diese Teilaktivität in den kooperativen Schreibprozess einzubinden und für die Schülerinnen und Schüler durch die TAs zu modellieren.

Der vorliegende Einblick in die Arbeit der TAs legt für weitere Studien eine Ergänzung der ursprünglichen Fragestellungen nahe: Im Kapitel «Ergebnisdarstellung. Soziale Interaktion» wurde festgehalten, dass unter Anleitung und Beteiligung der TAs als Schreibcoaches ein Prozess stattfindet, bei dem davon auszugehen ist, dass er den Grundsätzen des kooperativen Schreibens (Lowry et al., 2004) folgt. Dieser Annahme kann an dieser Stelle nicht widersprochen werden, aber es wäre notwendig, Zusammenhänge zwischen den Vorgaben eines Schreibprojekts, dem Selbstverständnis der TAs und der Ausgestaltung des kooperativen Schreibprozesses deutlicher zu machen. Die Aussagen der an

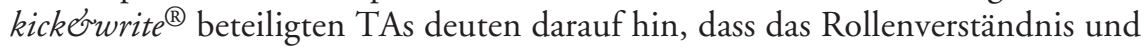
die "hybrid identity» (Rabkin et al., 2011) für die Ausgestaltung des kooperativen Schreibprozesses entscheidend sind. Um diesen Zusammenhängen auf den Grund zu gehen, müssten die TAs vertieft befragt werden. Gruppenstrategien und Formen der Zusammenarbeit bei einzelnen Aktivitäten von 
der Ideensammlung bis zur Endredaktion müssten genau erfasst werden. Die Datenbasis wäre durch in vivo-Aufnahmen der mündlichen Kommunikation zwischen den Beteiligten während des Schreib- und Beurteilungsprozesses und eine Sammlung der Entwürfe und Textbausteine aus den verschiedenen Arbeitsstadien zu ergänzen. Solche Studien auf breiter Datenbasis wären angesichts der noch kaum vorhandenen Forschung zur Arbeit des TA im deutschsprachigen Raum wünschenswert. Die Ergebnisse könnten in die Aus- und Weiterbildung interessierter Autorinnen und Autoren einfliessen, die Einsätze als TA in Betracht ziehen.

\section{Anmerkung}

1 Die beiden ausgewählten Teaching Artists haben sich in ihrem Vorgehen am deutlichsten unterschieden - einer (Kickfuchs) liess den entstehenden Text von den Schülerinnen und Schülern schreiben, der andere (Ballfee) formulierte die Ideen der Kinder für den Roman selber zum Text aus. Gleichzeitig haben sich die beiden ausgewählten Teaching Artists aus schreibdidaktischer Sicht am prägnantesten und elaboriertesten geäussert.

\section{Literaturverzeichnis}

Alves, K., Blume, B., Bortlik, W. \& Hughes, S. (2014). Vier verrückte Fussballgeschichten. Solothurn: kwasi.

Artlink Büro für Kulturkooperation. (2017). Autorin in Schulresidenz. Zugriff unter http:// www.artlink.ch/index.php?pageid $=140 \&$ lang $=\mathrm{d}$

Bachmann, T. \& Becker-Mrotzek, M. (2010). Schreibaufgaben situieren und profilieren. In T. Pohl \& T. Steinhoff (Hrsg.), Textformen als Lernformen (S. 191-209). Duisburg: Gilles $\&$ Francke.

Booth, E. (2003). From the Editor: Welcome to the Teaching Artist Journal - the national forum that seeks to clarify, enrich and advance Teaching Artist practice. Teaching Artist Journal, 1, 3-4. doi:10.1080/15411796.2003.9684264

Booth, E. (2009). The Music Teaching Artists Bible. Oxford: Oxford University Press.

Bürki, G. \& Stucki-Volz, G. (2010). "Zu zweit allein» - kooperatives Schreiben am Beispiel eines Klassenromans. Diskussion und Reflexion eines schulischen Schreibprojekts. Der Deutschunterricht, 6, 89-95.

Claussen, C. \& Merkelbach, V. (1995). Erzählwerkstatt. Mündliches Erzählen. Braunschweig: Westermann.

Gramespacher, E., Gyger, M., Supino, F., Beckert, Ch., Koch, P. \& Weigel, P. (2015). kick\&write ${ }^{\circledR} 2014$ - An Interdisciplinary Project in Swiss Primary Schools. eská kinantropologie (Czech Kinanthropology), 19, (4), 23-33.

Hochschule der Künste Bern (HKB) (2017). CAS Teaching Artist. Zugriff unter https:// www.hkb.bfh.ch/de/weiterbildung/kulturvermittlung/cas-teaching-artist/

Kulturvermittlung Kanton Aargau (2107). Kultur macht Schule. Zugriff unter https://www. ag.ch/de/bks/kultur/kulturvermittlung/kultur_macht_schule_1/kulturmachtschule.jsp

Leubner, M., Saupe, A. \& Richter, M. (2010). Literaturdidaktik. Berlin: Akademie-Verlag.

Lowry, P.B., Curtis, A. \& Lowry, M.R. (2004). Building a Taxonomy and Nomenclature of Collaborative Writing to Improve Interdisciplinary Research and Practice. Journal of Business Communication, 41, 66-99.

Mayring, Ph. (2003). Qualitative Inhaltsanalyse. Grundlagen und Techniken. Weinheim \& Basel: Beltz. 
Rabkin, N., Reynolds, M., Hedberg, E. \& Shelby, J. (2011). Teaching Artists and the Future of Education. A Report on the Teaching Artist Research Project. Chicago, IL: National Opinion ResearchCenter(NORC). Retrieved fromhttp://www.norc.org/PDFs/TARP\%20Findings/ Teaching_Artists_Research_Project_Final_Report_\%209-14-11.pdf

Die Provinz, Kulturprojekte (2017). Schulhausroman. Zugriff unter http://www.provinz.ch/ Rosebrock, C. \& Bertschi-Kaufmann, A. (2013). Literalität erfassen: bildungspolitisch, kulturell, individuell. In. C. Rosebrock \& A. Bertschi Kaufmann (Hrsg.), Literalität erfassen: bildungspolitisch, kulturell, individuell (S. 7-12). Weinheim und Basel: Beltz Juventa.

Volksschulamt Kanton Zürich (2017). Literatur aus erster Hand. Zugriff unter http://www. schuleundkultur.zh.ch/537.0.html

Schlagworte: Teaching Artist, Primarschule, Literalität, Motivation, kooperatives Schreiben

\section{"Représentez-vous ça dans votre tête» - Comment des artistes écrivent avec des élèves d'école primaire}

\section{Résumé}

En 2014, juste avant la Coupe du Monde de football, quatre auteurs reconnus sont intervenus en tant que Teaching Artists dans des classes de l'école primaire du nord-ouest de la Suisse. Ils ont animé des ateliers d'écriture dans le cadre du projet kickorwrite@. Leur objectif était de motiver des enfants, dont la socialisation à la littératie était faible, à lire et à écrire en les incitant à rédiger un récit portant sur le football. Le recours à des Teaching Artists jouant le rôle de coachs de l'écriture est une mesure d'encouragement spéciale à la littératie. Cet article présente les objectifs et les concepts artistiques et didactiques (didactique de l'allemand) des Teaching Artists et documente leur perception du processus de création des enfants. Les résultats montrent une augmentation de la motivation à la lecture, grâce, notamment, à un accès inhabituel à l'écriture dans un lieu d'apprentissage informel, à la possibilité d'exprimer des compétences au-delà des normes purement linguistiques, à une communication sur les textes et à un accompagnement intensif.

Mots-clés: Teaching Artist, école primaire, littéracie, motivation, écriture collaborative 


\section{"Cercate di farvi una rappresentazione mentale" - Come autori (teaching artists) scrivono con allievi di scuola elementare}

\section{Riassunto}

Nell'anno 2014 nei mesi precedenti al Campionato Mondiale di calcio in Brasile quattro autori rinomati hanno agito da Teaching Artists in quattro classi di differenti scuole elementari della Svizzera nordoccidentale. Le autrici e l'autore hanno condotto nell'ambito del progetto kick\&write ${ }^{\circledR}$ dei seminari di scrittura in classi residenti in aree svantaggiate con l'obiettivo di motivare i ragazzi alla lettura e alla scrittura, incitandoli a redigere un testo sul calcio. La collaborazione con dei teaching artist è un provvedimento didattico per favorire il letteratismo degli alunni coinvolti. L'articolo descrive le mete artistiche e didattiche del progetto e $\mathrm{i}$ diversi concetti adottati dai teaching artist nell'imposizione del loro lavoro creativo con gli alunni. La motivazione degli allievi per la lettura è aumentata, grazie all'inconsueto approccio alla scrittura in un luogo d'apprendimento informale, alla possibilità di vivere competenze al di là di norme prettamente linguistiche, alla comunicazione che seguiva alle attività letterarie e ad un'intenso accompagnamento.

Parole chiave: Teaching Artist, scuola elementare, letteratismo, motivazione, scrittura collaborative

\section{"Imagine in your head" - How Teaching Artists write with primary school students}

\section{Abstract}

In the run-up to the football World Cup 2014, four established authors acted as Teaching Artists in primary school classes in Northwestern Switzerland. In the project kickowrite ${ }^{\circledR}$ 2014, they led writing workshops which aimed at motivating children with an unfavorable literacy socialization to read and write. Their task was to write a text on football. To appoint Teaching Artists as writing coaches is a special school support measure in the area of literacy. This contribution specifies the artistic and educational (German didactics) aims and concepts of the Teaching Artists and documents their perception of the children's creative process. In this place of informal education, reading motivation increased, because of an unusual access to literacy, the experience of competency beyond formal norms of language, communication linked to these literary activities, and intensive support.

Keywords: Teaching Artist, primary school, literacy, motivation, collaborative writing 